\title{
Recurrent Hepatic Inflammatory Pseudotumor in Asynchronism After Surgery of Inflammatory Pseudotumor Associated With Granulomatous Cholangitis
}

\author{
Akira Imoto $^{\mathrm{a}, \mathrm{d}}$, Yoshihiro Inoue ${ }^{\mathrm{b}}$, Takeshi Ogura ${ }^{\mathrm{a}}$, Daisuke Masuda ${ }^{\mathrm{a}}$, Mitsuhiro Asakuma ${ }^{\mathrm{a}}$, \\ Yoshitaka Kurisu $^{\mathrm{c}}$, Kazuhisa Uchiyama ${ }^{\mathrm{b}}$, Kazuhide Higuchi ${ }^{\mathrm{a}}$
}

\begin{abstract}
A 64-year-old man was referred to our hospital for investigation of thickening of the gallbladder wall and hepatic mass. Repeated endoscopic ultrasound-guided fine-needle aspiration examinations of these lesions excluded malignant diseases. Cholecystectomy and resection of lateral section confirmed xanthogranulomatous cholecystitis and inflammatory pseudotumor (IPT) associated with granulomatous cholangitis. Ten months after surgery, a new lesion appeared in the liver medial section, associated with fluorodeoxyglucose accumulation. After performing resection of the affected segment, recurrent IPT related with granulomatous cholangitis was diagnosed. No subsequent recurrence was seen during follow-up. This represents the first report of recurrent hepatic IPT originating from granulomatous cholangitis after resection of xanthogranulomatous cholecystitis and IPT caused by granulomatous cholangitis.
\end{abstract}

Keywords: Inflammatory pseudotumor; Granulomatous cholangitis: Recurrence; Surgery

\section{Introduction}

Inflammatory pseudotumor (IPT) is a rare benign condition reported in almost all organs, including occasionally in the liver [1]. IPT was first described in the lung in 1939 and liver involvement was first described in $1953[2,3]$. In a study of focal lesions resected from 403 patients, the incidence of hepatic IPT was reported as $0.7 \%$ [4]. The etiology and pathogenesis of IPT remain uncertain, although infection, autoimmune phe-

Manuscript submitted December 30, 2018, accepted January 14, 2019

aSecond Department of Internal Medicine, Osaka Medical College, Takatsuki, Osaka, Japan

bDepartment of General and Gastroenterological Surgery, Osaka Medical College, Takatsuki, Osaka, Japan

'Department of Pathology, Osaka Medical College, Takatsuki, Osaka, Japan

${ }^{\mathrm{d} C}$ Corresponding Author: Akira Imoto, Second Department of Internal Medicine, Osaka Medical College, 2-7 Daigaku-machi, Takatsuki-shi, Osaka 5698686, Japan. Email: akiraimoto27@gmail.com

doi: https://doi.org/10.14740/jmc3245 nomena, and systemic inflammatory response syndrome have been suggested as possible causes [5-7]. IPT of the liver is often difficult to differentiate from other malignant diseases, as the incidence is rare, the pathogenesis is diverse, and imaging findings are variable [8-11]. The treatment of choice is still surgical resection. There is always a possibility of recurrence with IPT, but few reports have described recurrence after resection. Here, we describe the case of a patient with IPT of the liver associated with granulomatous cholangitis after resection of xanthogranulomatous cholecystitis and IPT caused by granulomatous cholangitis.

\section{Case Report}

A 65-year-old man was referred to our hospital for detailed investigations of abnormalities of the gallbladder and left lobe of the liver detected incidentally on computed tomography (CT) performed during follow-up for angina. The patient had received treatment at a local doctor for hypertension, diabetes mellitus, dyslipidemia, and angina, and had no history of surgery. He was a smoker (20 cigarettes/day for 40 years) and drank occasionally.

On examination, axillary temperature was $36.5^{\circ} \mathrm{C}$, heart rate was 63 beats/min, blood pressure was 109/49 $\mathrm{mm} \mathrm{Hg}$, and respiratory rate was 16 breaths/min. Laboratory tests showed a mild inflammatory response with no biochemical abnormalities. Serum levels of carcinoembryonic antigen, $\alpha$-fetoprotein, carbohydrate antigen 19-9, and protein induced by vitamin $\mathrm{K}$ absence or antagonist 2 were not elevated.

CT revealed an irregularly thickened gallbladder (GB) wall with an indistinct boundary with the neighboring liver (Fig. 1a) and an irregularly ring enhanced, hypointense area in the left lobe of the liver (Fig. 1b), both of which showed accumulation of fluorodeoxyglucose (FDG) on positron emission tomography (PET) (Fig. 1c, d). On endoscopic ultrasound (EUS), the Rokitanski-Aschoff sinus with stones was seen on the GB wall and the borderline between the GB and liver was unclear (Fig. $2 a)$. The mass in the left lobe was visualized as an aggregation of highly echoic spots (Fig. 2b). Repeated EUS-guided fine-needle aspiration (EUS-FNA) for GB wall and the mass in the left lobe excluded malignant diseases (Fig. 2c-e). Although findings from CT were suggestive of GB cancer and liver metastasis, EUS and EUS-FNA findings suggested these lesions represented xanthogranulomatous cholecystitis and IPT caused 




Figure 1. CT reveals an irregularly thickened GB wall showing an indistinct boundary with the neighboring liver (a) and an irregular, ring-enhancing, hypointense area in the left lobe of the liver (b). Both lesions show FDG accumulation on PET (c and d).

by granulomatous cholangitis. Under the above provisional diagnosis, cholecystectomy and lateral sectional resection of the liver were performed 3 months after these examinations. Foam cells and multinucleated giant cells were seen with xanthogranulomatous inflammation in the GB wall (Fig. 3a, b), and similar results were seen for a nearby bile duct in the liver (Fig. 3c, d), pathologically confirming the lesions as xanthogranulomatous cholecystitis and granulomatous cholangitis, respectively. The cause of granulomatous cholangitis, such as biliary infection or biliary obstruction, was not found pathologically. Immunohistochemical studies showed negative staining for immuno- globulin (Ig) G4 (data not shown). No fungi or acid-fast bacilli were found on periodic acid-Schiff or Ziehl-Neelsen staining. Inflammatory infiltrations were not found in the edge of the specimens of the liver. On follow-up 10 months postoperatively, CT showed a new, irregular mass in the liver medial section, showing delayed enhancement and accumulation of FDG on PET (Fig. 4). Although the lesion was considered to most likely represent recurrent IPT caused by granulomatous cholangitis, malignant disorder was not able to be completely excluded. The patient underwent liver medial sectional resection, and pathological examination confirmed granulomatous inflammation,
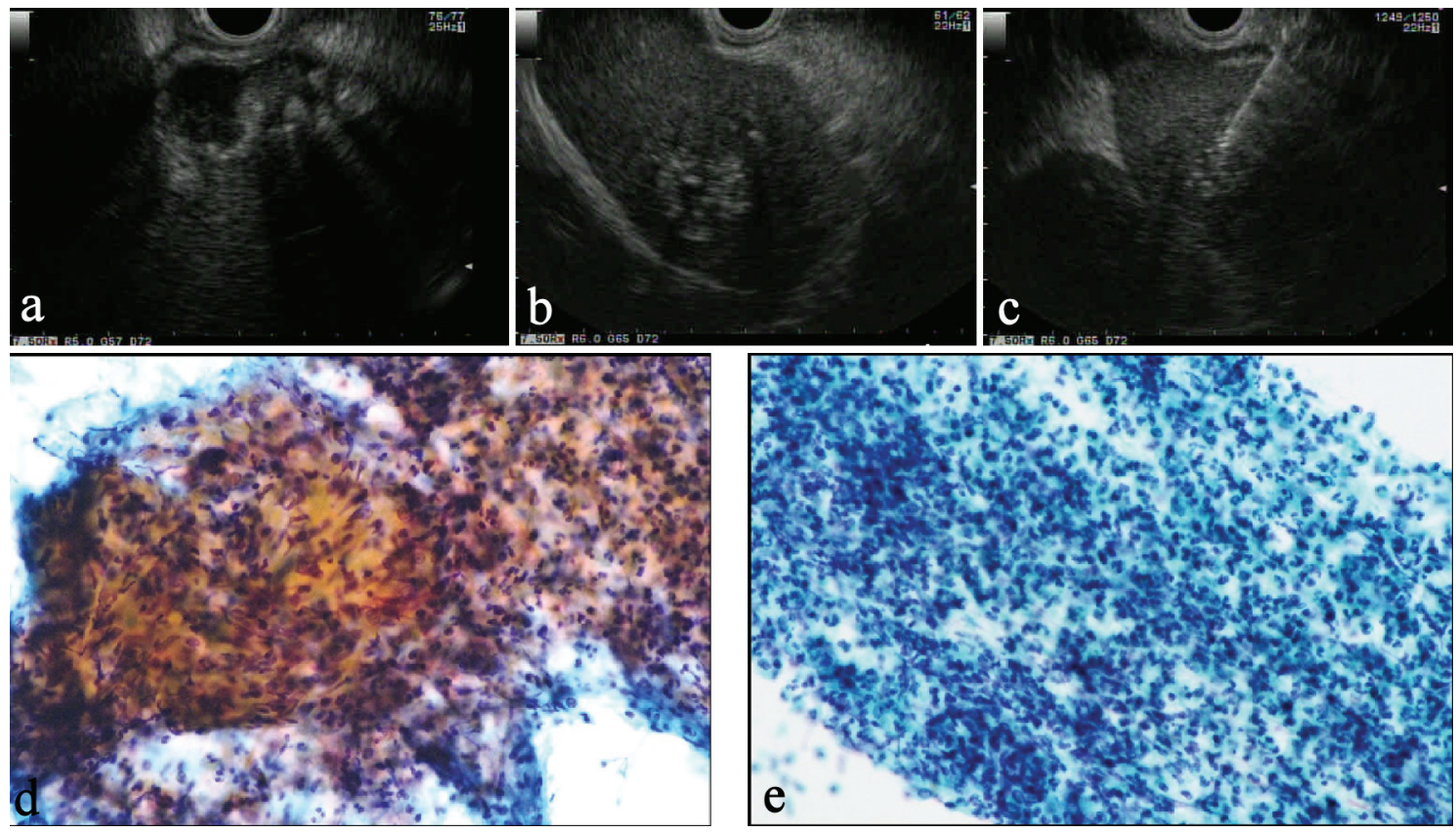

Figure 2. (a) On EUS, a Rokitansky-Aschoff sinus with stones is seen in the GB wall and the border between the GB and liver is unclear. (b) The mass in the left lobe is visualized as an aggregation of highly echoic spots. (c) EUS-FNA reveals typical inflammatory cells from both the liver mass (d) and GB wall (e), suggesting these lesions were not malignant disorder. 

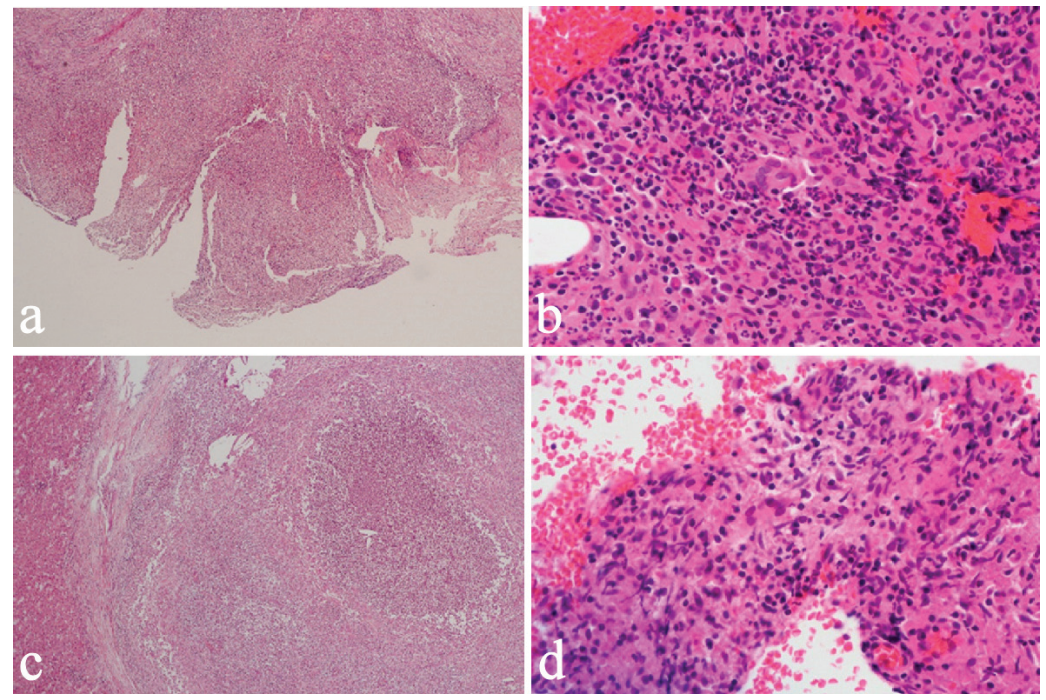

Figure 3. Cholecystectomy and liver lateral sectional resection showed foam cells and multinucleated giant cells with xanthogranulomatous inflammation in the GB wall $(a, b)$ and similar phenomena in a nearby bile duct in the liver (c, d).

multinucleated giant cells and neutrophilic infiltration with fibrosis around the bile duct (Fig. 5a, b), consistent with the features of granulomatous cholangitis. The final diagnosis was metachronous recurrence of IPT associated with granulomatous cholangitis. While further recurrence remains possible in the future, the patient has shown no additional recurrence as of the time of writing, 36 months after the second surgery.

\section{Discussion}

A study by Zen et al classified hepatic IPT into two types: fi- brohistiocytic type; and lymphoplasmacytic type [7]. Fibrohistiocytic IPT was associated with granulomatous inflammation and multinucleated giant cells, while lymphoplasmacytic IPT was histologically characterized by diffuse inflammatory cell infiltration, mainly comprising lymphocytes and plasma cells. The current case involved displayed inflammatory cells, granulomatous and multinucleated giant cells, consistent with fibrohistiocytic IPT. These histological features were observed in both GB and mass of the lateral segment. Because the etiology of granulomatous cholangitis in liver was unclear even with pathological evaluation, it was unknown whether there was a relationship between GB lesion and mass in the lateral
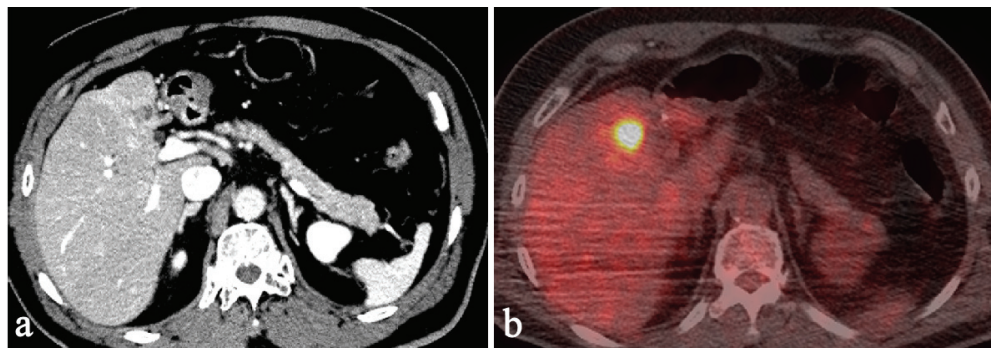

Figure 4. Ten months after first surgery, follow-up CT shows a new, irregular mass in the liver medial section. The mass shows
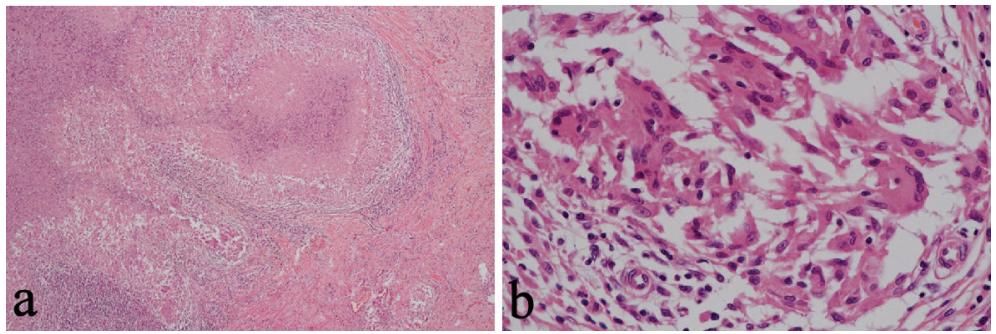

Figure 5. Following liver medial sectional resection, pathological examination revealed granulomatous inflammation, multinucleated giant cells and neutrophilic infiltration with fibrosis around a bile duct $(a, b)$. 
segment in the present case. Although it could not be proved pathologically, the simultaneous rise of internal pressure of the bile duct and gallbladder due to some cause such as infection and obstruction of the bile duct was possible.

Methods of obtaining histological tissue from the liver such as liver biopsy and EUS-FNA carry a risk of needle-tract seeding in patients with malignant tumors $[12,13]$. In the present case, the mass in the left lobe of the liver resembled a metastasis from a GB cancer on CT at the time of the first surgery. Even if the GB lesion had represented cancer, seeding would not represent a priority problem if metastasis has already occurred. However, biopsy was not performed in consideration of seeding at the time of the second surgery. EUS-FNA was useful to exclude malignant disease at the first surgery, but tumor biopsy is not necessary when planning surgical interventions for the liver.

The optimal treatment for hepatic IPT has yet to be established $[14,15]$. Due to its ambiguity, the lesion completely resolves in some patients receiving antibiotics and/or corticosteroids, but some lesions recur [15-17]. Numerous studies have suggested surgery as the treatment of choice, particularly for patients with severe symptoms or an indeterminate diagnosis $[1,18,19]$.

The cause of IPT recurrence in the present case might be the persistence of inflammation associated with xanthogranulomatous cholecystitis. The existence of inflammation around fundus of GB on CT obtained just before first surgery (data not shown) and the near distance between GB and recurred position supported this idea. Seeing from this point of view, surgery should have been delayed until inflammation was relieved a little more on CT. If a similar hepatic IPT appears in the future in this patient, observation may be appropriate, because the IPT may be benign and the risk entailed in further resection of the remnant liver may be too high. At such a time, EUS-FNA for the IPT may be acceptable to exclude malignancy. At present, no sign of recurrence has been seen as of 36 months after the second surgery.

In conclusion, recurrent hepatic IPT associated with granulomatous cholangitis is a rare condition. EUS-FNA was useful for achieving an accurate diagnosis and preventing misdiagnosis as malignant tumor.

\section{Acknowledgments}

We would like to thank Dr. Etsuko Makino, Setsuko Tsujimura, Mayumi Hagiwara, and Toru Imoto for their helpful suggestions regarding the manuscript.

\section{Financial Disclosures}

There are no financial relationships to disclose for any of the authors.

\section{Abbreviations}

GB: gallbladder; EUS: endoscopic ultrasound; EUS-FNA: endoscopic ultrasound-guided fine-needle aspiration; IPT: inflammatory pseudotumor; CT: computed tomography; FDG: fluorodeoxyglucose; PET: positron emission tomography; CD: cluster of differentiation; Ig: immunoglobulin

\section{References}

1. Bae SK, Abiru S, Kamohara Y, Hashimoto S, Otani M, Saeki A, Nagaoka S, et al. Hepatic inflammatory pseudotumor associated with xanthogranulomatous cholangitis mimicking cholangiocarcinoma. Intern Med. 2015;54(7):771-775.

2. Copin MC, Gosselin BH, Ribet ME. Plasma cell granuloma of the lung: difficulties in diagnosis and prognosis. Ann Thorac Surg. 1996;61(5):1477-1482.

3. Pack GT, Baker HW. Total right hepatic lobectomy; report of a case. Ann Surg. 1953;138(2):253-258.

4. Torzilli G, Inoue K, Midorikawa Y, Hui AM, Takayama T, Makuuchi M. Inflammatory pseudotumors of the liver: prevalence and clinical impact in surgical patients. Hepatogastroenterology. 2001;48(40):1118-1123.

5. Horiuchi R, Uchida T, Kojima T, Shikata T. Inflammatory pseudotumor of the liver. Clinicopathologic study and review of the literature. Cancer. 1990;65(7):1583-1590.

6. White JE, Chase CW, Kelley JE, Brock WB, Clark MO. Inflammatory pseudotumor of the liver associated with extrahepatic infection. South Med J. 1997;90(1):23-29.

7. Zen Y, Fujii T, Sato Y, Masuda S, Nakanuma Y. Pathological classification of hepatic inflammatory pseudotumor with respect to IgG4-related disease. Mod Pathol. 2007;20(8):884-894.

8. Honmyo N, Kobayashi T, Tashiro H, Ishiyama K, Ide K, Tahara H, Ohira M, et al. Inflammatory pseudotumor of the liver occurring during the course of hepatitis $\mathrm{C}$ virusrelated hepatocellular carcinoma treatment: A case report. Int J Surg Case Rep. 2016;20:96-100.

9. Matsuo Y, Sato M, Shibata T, Morimoto M, Tsuboi K, Shamoto T, Hirokawa T, et al. Inflammatory pseudotumor of the liver diagnosed as metastatic liver tumor in a patient with a gastrointestinal stromal tumor of the rectum: report of a case. World J Surg Oncol. 2014;12:140.

10. Kai K, Matsuyama S, Ohtsuka T, Kitahara K, Mori D, Miyazaki K. Multiple inflammatory pseudotumor of the liver, mimicking cholangiocarcinoma with tumor embolus in the hepatic vein: report of a case. Surg Today. 2007;37(6):530-533.

11. Ke Q, Fan L, Duan X, He Z, Zheng S. Inflammatory pseudotumor mimicking primary hepatic malignant tumor with hepatitis B virus-related cirrhosis: A case report. Oncol Lett. 2013;6(2):550-552.

12. Zhong-Yi Z, Wei Y, Kun Y, Ying D, Wei W, Jung-Chieh L, Min-Hua C. Needle track seeding after percutaneous radiofrequency ablation of hepatocellular carcinoma: 14year experience at a single centre. Int J Hyperthermia. 2017;33(4):1-8.

13. Iida T, Adachi T, Ohe Y, Nakagaki S, Yabana T, Kondo Y, Nakase H. Re-recurrence after distal gastrectomy for recurrence caused by needle tract seeding during endoscopic ultrasound-guided fine-needle aspiration of a pancreatic 
adenocarcinoma. Endoscopy. 2016;48(S 01):E304-E305.

14. Di Vita G, Soresi M, Patti R, Carroccio A, Leo P, Franco V, Montalto G. Concomitant inflammatory pseudotumor of the liver and spleen. Liver. 2001;21(3):217-222.

15. Salakos C, Nikolakopoulou NM, De Verney Y, Tsamandas AC, Ziambaras T, Petsas T, Papanastasiou DA. Anaplastic lymphoma kinase (ALK) positive inflammatory pseudotumor of the liver: conservative treatment and long-term follow-up. Eur J Pediatr Surg. 2010;20(4):278280 .

16. Yamaguchi J, Sakamoto Y, Sano T, Shimada K, Kosuge T. Spontaneous regression of inflammatory pseu- dotumor of the liver: report of three cases. Surg Today. 2007;37(6):525-529.

17. Hamzaoui L, Medhioub M, Mahmoudi M, Chelbi E, Bouzaidi K, Msadak Azouz M. Inflammatory pseudotumor of the liver. Presse Med. 2016;45(9):804-807.

18. Koea JB, Broadhurst GW, Rodgers MS, McCall JL. Inflammatory pseudotumor of the liver: demographics, diagnosis, and the case for nonoperative management. J Am Coll Surg. 2003;196(2):226-235.

19. Zhang Y, Lu H, Ji H, Li Y. Inflammatory pseudotumor of the liver: A case report and literature review. Intractable Rare Dis Res. 2015;4(3):155-158. 